\title{
A Controlled Experiment Investigating the Effects of Explanatory Manual on Adherence to Operating Procedures
}

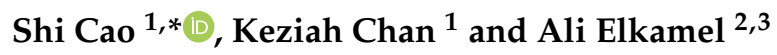 \\ 1 Department of Systems Design Engineering, University of Waterloo, Waterloo, ON N2L 3G1, Canada; \\ kn2chan@uwaterloo.ca \\ 2 Department of Chemical Engineering, University of Waterloo, Waterloo, ON N2L 3G1, Canada; \\ aelkamel@uwaterloo.ca \\ 3 Department of Chemical Engineering, Khalifa University, Abu Dhabi 127788, United Arab Emirates \\ * Correspondence: shi.cao@uwaterloo.ca; Tel.: +1-519-888-4567
}

Received: 20 February 2019; Accepted: 9 April 2019; Published: 14 April 2019

\begin{abstract}
Operators' adherence to operating procedures is a crucial factor for process safety in the process industry. Instruction manuals that document Standard Operating Procedures (SOP) are commonly used both as training materials and references during operation. Traditional SOP manual design emphasized using simple step-by-step instructions for how to do the tasks, but it often neglected the reasons why the steps and their specific orders should be closely followed. It is evident that operators sometimes choose to deviate from SOP intentionally if they do not understand the reasons and incorrectly deem the steps in the manual as slow or outdated. To help bridge the knowledge gap between SOP designers and operators, we advocate explanatory SOP manual design that adds the reasons for the steps in manual instructions. To examine the effect of explanatory manual, we conducted a controlled experiment using a hydraulic pump system that represented the wash operation in the electroplating industry. Participants' performance and adherence to operating procedures (both Adherence to Production Order Procedures and Adherence to Wait Time) were measured and compared between the explanatory manual and the procedural manual conditions. The results showed that the explanatory manual had the benefit of increasing Adherence to Production Order Procedures, while time performance, Percent Duration within Bounds, and Adherence to Wait Time were not significantly affected. The finding supports the use of explanatory manuals because they have the potential to serve as an effective and economic way to improve operators' adherence to operating procedures and process safety. Limitations of the laboratory setup were discussed.
\end{abstract}

Keywords: standard operating procedures; manual design; adherence to operating procedures; process safety; operator performance

\section{Introduction}

Industrial operations must follow proper procedures to ensure safety, productivity, and quality. Most organizations use Standard Operating Procedures (SOPs) that document official instructions for operators to follow. The application of SOP reflects the knowledge and skills from both procedure designers and operators. As discussed by Dien [1], the optimal distribution of responsibility between designers and operators depends on the complexity and variability in the operation. For complex operations, such as diagnosing a system error that could have many different causes, it is often difficult for designers to provide very specific procedures covering all the situations (Work-As-Imagined). In such cases, it is important to understand operators' capability and flexibility to make things go right (Work-As-Done), as advocated by Hollnagel, Wears, and Braithwaite in the Safety-II approach [2]. 
In complex work domains, such as aviation and healthcare, greater responsibility resides with the operators who need to apply their knowledge in identifying the situation and solving the problem. Sometimes, operators choose not to adhere to SOP intentionally because they think of SOP as outdated, less efficient, or not applicable to the specific case. For example, a field study of nuclear power plant operators found that operators frequently made decisions using their experience and knowledge rather than strictly following SOP when dealing with micro-incidents [3]. A report of forensic analysts also showed that analysts often treat SOP as a baseline of knowledge that informs practice and they often rely on tips from colleagues and personal experience [4].

On the other hand, for simple routine operations, it is more likely for designers to provide an optimal SOP that covers detailed actions to carry out the tasks. In such cases, operators are expected to closely follow the instructions without deviation. Operator deviation from SOP has caused serious accidents. In 2005, the BP Texas City Refinery explosion killed 15 people and injured another 180 in the United States [5]. In this case, operators deviated from start-up SOP and continuously pumped flammable liquid hydrocarbons into the raffinate splitter tower of the unit for over three hours with no output. Chemical Safety and Hazard Investigation Board found that the operators developed their own practices to resolve reoccurring issues because they deemed the SOP outdated and ineffective $[5,6]$.

In another case of the Japanese Tokaimura nuclear accident in 1999 [7], operators deviated from SOP of uranium processing that prepares fuel for a nuclear reactor. Two workers were killed, and hundreds were exposed to nuclear radiation. The SOP required radioactive substance containing uranium to be gradually mixed and prepared in small amount to avoid dangerous radioactive chain reaction. However, the operators intended to save time and bypassed the procedure. They manually poured seven buckets of uranyl nitrate solution altogether into the precipitation tank, which created a concentration of uranium exceeding the critical mass of self-sustaining chain reaction.

In both the above cases, operators failed to follow SOP. It is important to note that the reason is not because they accidentally forgot the SOP. They knew the SOP but wanted to improve the procedures and be creative. However, they did not fully understand the mechanism of the process, nor the reasons for the critical steps in the SOP.

The lack of adherence to procedures is generally regarded as a problem in routine operation of the process industry. Researchers in the field of safety also emphasized the importance of studying operator adherence to procedures as an indicator of process safety [8,9]. How to effectively improve adherence to operating procedures remains a research question. Common practices have focused on operator re-training. If operator deviation is identified in the workplace, a common practice is to re-train the operators by requiring them to study the SOP again. However, a report in the pharmaceutical industry suggested that this common practice is not effective in reducing human error [10]. We argue that there is a problem in the current practice of SOP and instruction manual design.

SOP is often presented in written formats such as manuals. Manuals are used both as training materials and references in routine operation. For example, a typical manual for an oil refinery plant includes procedures for plant start-up, continuous operation, and shutdown. SOP designers are usually experts in the field and have more knowledge about the process than operators. When designing SOP, designers have their reasons for each step, each parameter, and the specific order of steps. Although SOP should inform operators both how to do the task step-by-step and why the steps must be followed, many SOP manuals and instructions focused on how, but were weak on why [11]. It is often recommended that SOP manuals should include "simple, step-by-step instructions that an operator who has basic knowledge can perform without supervision" [12]. Although sections describing warnings and cautions are also required in SOP manuals, such sections usually discuss the whole process in general, and there is a lack of explanation pertaining to each step of the procedure [13]. SOP designers may assume that operators should have the basic knowledge from their training and education to understand the reasons behind each step, but companies have the motive to reduce budget in hiring highly educated workers and training operators. In both the BP Texas City Refinery 
accident [5] and the Tokaimura nuclear accident [7], the operators did not have the knowledge needed for judging the situation nor inventing new procedures.

In this paper, we advocate a new approach to SOP manual design called explanatory manual. Explanatory manual design aims to bridge the knowledge gap between SOP designers and operators. SOP designers are experts of the process, but operators' role does not and should not require the same level of expertise. An explanatory manual concisely explains the reason behind each critical step of SOP, considering operators' knowledge background and tendency to simplify and deviate from SOP. Explanatory manuals can be used as guidelines and references during operation. They can also be used in training to emphasize safety knowledge pertaining to each step. Psychology studies showed that understanding the meaning of learning materials improves memory recall [14] because declarative memory is stored in a semantic network of concepts, and comprehension provides more meaningful concepts to be connected in the network for a stronger memory structure [15]. We expect that explanatory manual can improve adherence to operating procedures and therefore improve process safety.

To examine the effect of explanatory manual on adherence to operating procedures, we conducted a controlled experiment that asked participants to operate a hydraulic pump system simulating the washing operation in the electroplating industry. We designed two versions of manuals for the same procedures. The explanatory version introduced both how to do the task step-by-step and why, whereas the procedural version introduced only how. Performance and adherence to operating procedures were compared between participants receiving the two versions of manuals.

\section{Methods}

\subsection{Apparatus and Tasks}

The experimental setup of the hydraulic pump system used in the current study is shown in Figure 1. Hydraulic pumps are prominently used across many industrial processes. The experimental setup was constructed to represent a small pilot plant system that could be found in an industrial setting. The underlying mechanism of hydraulic pump systems is fluid mechanics, which is a fundamental area of study in chemical engineering. The setup was designed to cover only basic fluid mechanic concepts of flow rate and pressure so that participants were able to understand them through training and practice in the study.

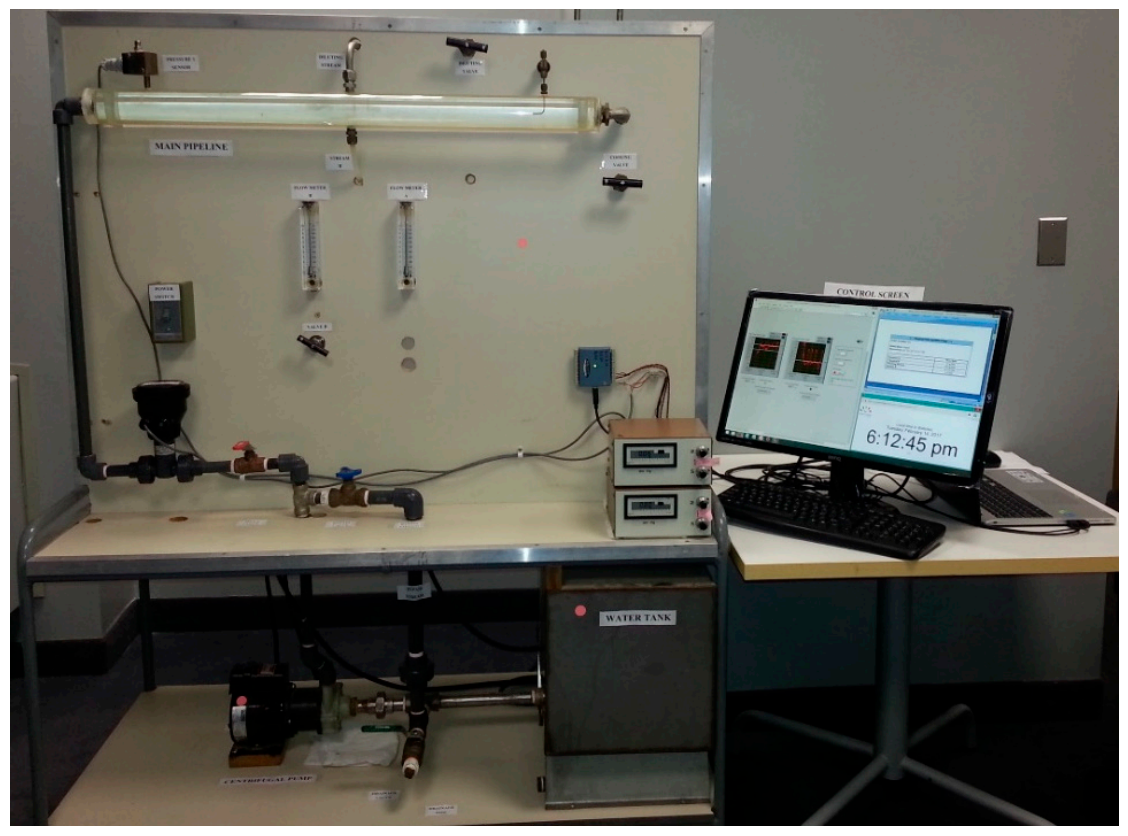

Figure 1. Experimental setup of the hydraulic pump system used in the current study. 
A schematic diagram of the hydraulic pump system is shown in Figure 2, which was also described to participants. It was a closed system that operated using a centrifugal pump to continuously pump water from the water tank to an elevated height, where it was then diverted from the main pipeline into a maximum of four streams (washing stream, stream B, diluting and cooling streams) and cycled back into the water tank. There were six valves in this system and participants were required to operated five of them. The inlet valve was used during start-up and shutdown. The bypass valve, valve B, diluting valve, and cooling valve were used to adjust operating criteria and control parameters within their required ranges. There were two operation parameters, washing stream flow rate and stream $B$ flow rate. Flow rate sensors and the LABVIEW software were used to measure and display flow rates. A computer was used to display the control screen, which consisted of chemical input controls (simulated), visual alarms, and operating criteria indicators. To ensure safety of the participants, only water was used in the pump system. Participants were instructed to imagine it as an industrial situation where chemicals were used.

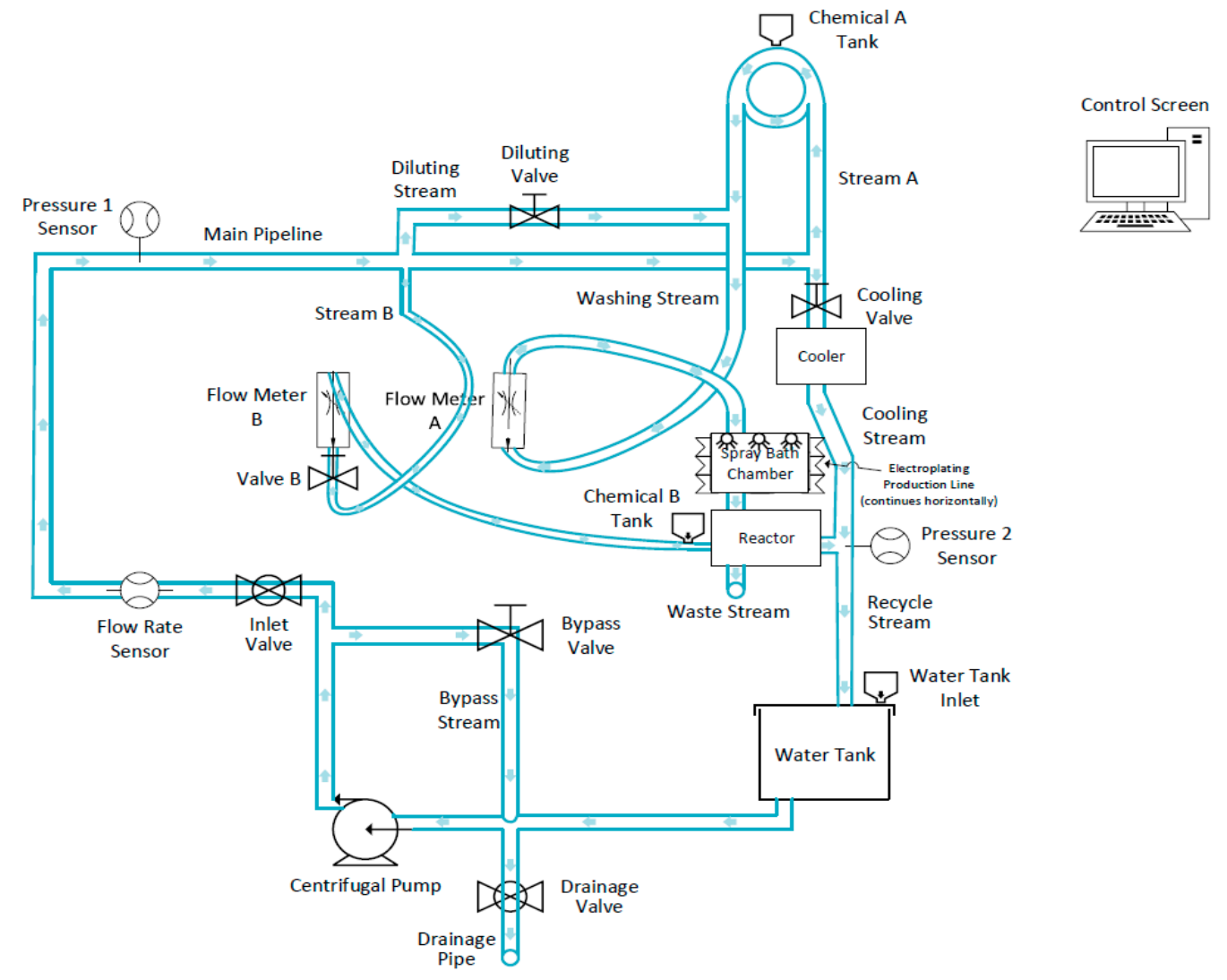

Figure 2. Schematic diagram of the experimental setup.

The experimental tasks required participants to operate the pump system in accordance to SOPs for start-up, continuous operation, and shutdown. The SOPs were explained in the manual provided to participants to learn and use. The experimental scenario assumed that the participant was hired as a new operator in charge of the wash system (i.e., the hydraulic pump system) at a metal manufacturing plant. The tasks were designed based on several common scenarios encountered during actual production in the process industry. First, participants started the pump system following start-up SOP. Then, during normal operation, they were instructed to always (1) maintain inlet flow rate within the range of 15-20 L/min and (2) maintain a pressure drop less than 2 psi. These two objectives represented the safety requirements in this experimental scenario. In addition to these basic requirements, each participant received four trials of production change tasks during normal operation. Each trial was initialized by a production order that instructed the participant to change stream B flow rate and washing stream flow rate to new values specified in the production order. They were also instructed to 
complete all changes made to the system as quickly as possible. The difficulty of this task was that participants had to manipulate four flow control valves to set four parameters within the required range, and all four valves influenced all four parameters. The first trial was treated as practice, and the results from the last three trials were included in analyses. Finally, participants shut down the system following shutdown SOP.

Regarding the two types of manuals, both procedural and explanatory manuals contained basic information including introduction of the scenario, electroplating process, wash system, operating criteria, production order, start-up steps, production change steps, and shutdown steps. The information was sufficient to operate the system properly if participants closely followed all the instructions. The explanatory manual had additional explanation of the purposes of the important steps, as shown in Tables 1-3 for the start-up, production change, and shutdown procedures respectively.

Table 1. Step-by-step instructions for the start-up procedure, as provided to the participants in the explanatory manual condition. The PURPOSE sections were only included in the explanatory manual condition but not in the procedural manual condition, while other words were the same.

\begin{tabular}{|c|c|}
\hline Step & Instruction \\
\hline 1 & $\begin{array}{l}\text { Ensure the power switch of the pump is turned off. Push the switch downwards to turn off } \\
\text { the power. }\end{array}$ \\
\hline 2 & $\begin{array}{l}\text { Ensure all six valves are fully closed (i.e., inlet, bypass, valve B, diluting, cooling and } \\
\text { drainage valves). Ball valves can be closed by turning it } 90 \text { degrees to the left. Alternatively, } \\
\text { flow control valves can be closed by turning it in a clockwise direction all the way. } \\
\text { PURPOSE: Fully close inlet valve at pump startup to induce a shut-off condition that can reduce } \\
\text { the initial load on the pump driver. }\end{array}$ \\
\hline 3 & Ensure the water tank is filled and the stopper is placed over the tank inlet. \\
\hline 4 & $\begin{array}{l}\text { Plug in the pump and sensors' electrical plugs into an electrical outlet. If using an } \\
\text { extension cord, ensure the power bar is switched on. }\end{array}$ \\
\hline 5 & Turn on the power switch of the pump by pushing the switch upwards. \\
\hline 6 & $\begin{array}{l}\text { After } 30 \mathrm{~s} \text {, gradually open the inlet valve by turning it } 90 \text { degrees to the right. } \\
\text { PURPOSE: Wait } 30 \text { s before opening inlet valve to ensure ample pump head is induced to minimize } \\
\text { the initial load on the pump driver and reduce cost. Beware that operation at zero flow for over } 1 \\
\text { min can cause pump failure. }\end{array}$ \\
\hline 7 & $\begin{array}{l}\text { Slightly open the diluting and bypass valves by turning them counterclockwise to get rid } \\
\text { of the air bubbles in the main pipeline. }\end{array}$ \\
\hline 8 & Check the entire wash system to ensure there are no leakages. \\
\hline 9 & $\begin{array}{l}\text { Wait for the system to reach steady state, as indicated by a constant inlet flow rate, and } \\
\text { maintain this condition for at least } 1 \text { min. } \\
\text { PURPOSE: Ensure all residues are flushed out and consistency of washing quality. }\end{array}$ \\
\hline
\end{tabular}

Table 2. Step-by-step instructions for the production change procedure, as provided to the participants in the explanatory manual condition. The PURPOSE sections were only included in the explanatory manual condition but not in the procedural manual condition, while other words were the same.

\begin{tabular}{|c|c|}
\hline Step & $\begin{array}{l}\text { Instruction } \\
\text { For Each New Production Order that is Manufactured, Use the Information Listed in Its } \\
\text { Production Notification (Refer to Notification System on the Control Screen) to Operate the } \\
\text { Wash System in Accordance with the Following General Procedure during Normal Operation: }\end{array}$ \\
\hline 1 & $\begin{array}{l}\text { Turn the cooling valve counterclockwise by } 1 \text { rotation to turn on the cooling stream. } \\
\text { PURPOSE: Preventative means to cool down temperature at the reactor exit to prevent build-up of heat to } \\
\text { dangerous levels. } \\
\text { At the same time, simultaneously turn the bypass and cooling valves as needed to maintain inlet } \\
\text { flow rate and pressure drop within specified operating criteria range. } \\
\text { PURPOSE: Ensures product quality and operation efficiency standards are satisfied. Note the relationship } \\
\text { between operating parameters. }\end{array}$ \\
\hline
\end{tabular}


Table 2. Cont.

\begin{tabular}{|c|c|}
\hline Step & $\begin{array}{l}\text { Instruction } \\
\text { For Each New Production Order that is Manufactured, Use the Information Listed in Its } \\
\text { Production Notification (Refer to Notification System on the Control Screen) to Operate the } \\
\text { Wash System in Accordance with the Following General Procedure during Normal Operation: }\end{array}$ \\
\hline 2 & $\begin{array}{l}\text { If current stream B flow rate < required stream B flow rate, slowly turn valve B counterclockwise to } \\
\text { increase flow rate of flow meter B. } \\
\text { If current stream B flow rate > required stream B flow rate, slowly turn valve B clockwise to decrease } \\
\text { flow rate of flow meter B. } \\
\text { PURPOSE: **Order of steps } 2-5 \text { is vital }{ }^{* *} \text { Set before inputting Chemical B to enable its transport once } \\
\text { inputted into the system. } \\
\text { At the same time, simultaneously turn the bypass and cooling valves as needed to maintain inlet } \\
\text { flow rate and pressure drop within specified operating criteria range. } \\
\text { PURPOSE: Ensures product quality and operation efficiency standards are satisfied. Note the relationship } \\
\text { between operating parameters. }\end{array}$ \\
\hline 3 & $\begin{array}{l}\text { Input the required mass flow rate of Chemical B onto the control screen. } \\
\text { PURPOSE: }{ }^{* *} \text { Order of steps } 2-5 \text { is vital }{ }^{* *} \text { Inputted prior to Chemical A to ensure that all Chemical A, } \\
\text { when added into the system, is removed from the recycle stream via reaction with Chemical B prior to being } \\
\text { sent back into the water tank. }\end{array}$ \\
\hline 4 & $\begin{array}{l}\text { If current washing stream flow rate < required washing stream flow range, slowly turn the diluting valve } \\
\text { counterclockwise to increase flow rate of flow meter A. } \\
\text { If current washing stream flow rate > required washing stream flow range, slowly turn the diluting valve } \\
\text { clockwise to decrease flow rate of flow meter A. } \\
\text { PURPOSE: }{ }^{* *} \text { Order of steps } 2-5 \text { is vital }{ }^{* *} \text { Set before inputting Chemical A to ensure Chemical A solution is } \\
\text { diluted to a suitable concentration once Chemical A is inputted into the system. } \\
\text { At the same time, simultaneously adjust valve B as needed to maintain flow rate of stream B at the } \\
\text { required rate, while also turning the bypass and cooling valves as needed to maintain inlet flow } \\
\text { rate and pressure drop within specified operating criteria range. } \\
\text { PURPOSE: Ensures product quality and operation efficiency standards are satisfied. Note the relationship } \\
\text { between operating parameters. }\end{array}$ \\
\hline 5 & Input the required mass flow rate of Chemical A onto the control screen. \\
\hline 6 & Ask the manager (the researcher) to come verify all operating parameters are met. \\
\hline 7 & $\begin{array}{l}\text { Maintain this condition for } 2 \text { min. } \\
\text { PURPOSE: Metal plates are washed in spray bath chamber for only } 1 \text { min (between 00:00:30 and 00:01:30). } \\
\text { However, maintaining steady state for } 2 \text { min (an extra } 30 \text { s before and after the metal plate enters the spray } \\
\text { bath chamber) ensures consistency of Chemical A solution and sufficient coverage of metal plate during } \\
\text { chamber entry and exit. }\end{array}$ \\
\hline 8 & Notify the manager that you have completed the production order. \\
\hline
\end{tabular}

Table 3. Step-by-step instructions for the shutdown procedure, as provided to the participants in the explanatory manual condition. The PURPOSE sections were only included in the explanatory manual condition but not in the procedural manual condition, while other words were the same.

\begin{tabular}{|c|c|}
\hline Step & Instruction \\
\hline 1 & $\begin{array}{l}\text { Operate an additional flush cycle of the system for } 1 \mathrm{~min} . \\
\text { PURPOSE: Ensure all remaining chemicals or contamination are flushed out. }\end{array}$ \\
\hline 2 & $\begin{array}{l}\text { Fully close the inlet valve by turning it } 90 \text { degrees to the left. } \\
\text { PURPOSE: Close the inlet valve so pump shutdown occurs at shut-off condition to reduce load on the } \\
\text { pump driver and reduce costs. }\end{array}$ \\
\hline 3 & Ensure the drainage valve remains closed. \\
\hline 4 & Turn off the power switch of the pump by pushing the switch downwards. \\
\hline 5 & $\begin{array}{l}\text { Unplug the pump and sensors' electrical plugs from the electrical outlet. If an extension cord } \\
\text { was used, switch off the power bar. }\end{array}$ \\
\hline
\end{tabular}


Table 3. Cont.

\begin{tabular}{cl}
\hline Step & \multicolumn{1}{c}{ Instruction } \\
\hline 6 & $\begin{array}{l}\text { Fully open valve B and the inlet, bypass, diluting and cooling valves to cycle all residual water } \\
\text { in the system back into the water tank by turning ball valves } 90 \text { degrees to the right and flow } \\
\text { control valves in a counterclockwise direction all the way. } \\
\text { PURPOSE: Ensure proper drainage of all streams back into the water tank. }\end{array}$ \\
\hline 7 & $\begin{array}{l}\text { Wait for the water in the main pipeline to fully drain out, then fully close all } 5 \text { valves by turning } \\
\text { ball valves } 90 \text { degrees to the left and flow control valves in a clockwise direction all the way. }\end{array}$ \\
\hline
\end{tabular}

\subsection{Experimental Design and Variables}

The experiment employed a $2 \times 3$ between-subject design. The primary independent variable was type of manual with two conditions, procedural manual and explanatory manual. Participants were randomly assigned to one of the two conditions. Since participants with different education background may have different levels of knowledge related to the hydraulic pump operation, participants' education level was recorded as a secondary independent variable, which included three conditions: Chemical engineering, other engineering, and other faculties (non-engineering). We also designed a test and tested participants' background knowledge about basic fluid mechanic concepts of hydraulic pump, flow rate, and pressure. The test score was recorded and included is the statistical analysis as a control variable.

The dependent variables included task completion time, Percent Duration within Bounds (PDWB), Adherence to Production Order Procedures (APOP), and Adherence to Wait Time (AWT).

Task completion time measured the average time duration in seconds required by a participant to complete a production change trial. It was measured by an experimenter using a stopwatch. Values from multiple trials of each participant were averaged for analysis.

Percent Duration Within Bounds (PDWB) measured the percentage of time duration that the operating criteria of inlet flow rate and pressure drop are met during production change trials. The values were calculated using LABVIEW.

Participants' adherence to operating procedures was measured in two different aspects. Adherence to Production Order Procedures (APOP) measured participants' degree of adherence to each step as well as the sequence of steps specified in SOP, with the exception of wait time procedures. Adherence to Wait Time (AWT) measured participants' degree of adherence to waiting for the full duration of the wait time specified in SOP. An incorrect action was defined as not waiting for the full duration. Both types of adherence are important for process safety. The BP Texas City Refinery explosion [5] exemplified the failure of APOP, while the Japanese Tokaimura nuclear accident [7] illustrated the failure of AWT. For both measures, a percentage score was calculated as the ratio of the total number of correct steps divided by the total number of steps. A survey question was also used at the completion of the experiment to ask participants for their reasons to deviate from SOP. The survey results were qualitatively analyzed to identify top reasons mentioned by the participants.

\subsection{Participants}

A total of 60 undergraduate and graduate students (31 male and 29 female) were recruited as participants from the University of Waterloo. Among them, 14 participants were from the department of chemical engineering, 18 from other engineering departments, and 28 from non-engineering departments. The study received ethics clearance through a University of Waterloo Research Ethics Committee. Participants received a remuneration of $\$ 30$ for their time. The study took about two hours for each participant. 


\subsection{Procedure}

Participants were given an information letter to read and completed the informed consent procedure. The information letter explained the general purpose of this study as studying operation manuals and operator performance but did not mention the two types of manuals nor the comparison between them. Since a between-subject design was used, each participant only experienced one type of manual, and the manuals contained no label of procedural or explanatory. Therefore, the participants' behavior was not likely to be affected by the researchers' hypothesis on the two types of manuals.

Participants completed a demographic survey and a background knowledge test about hydraulic pump operation and basic fluid mechanic concepts. Prior to the start of the formal experiment, participants were given an SOP manual (either the procedural or explanatory version) to read. Then, they started the formal experiment. Participants were instructed to operate the pump system closely following SOPs for start-up, continuous operation (with production change trials), and shutdown. After completing the tasks, they were asked to fill a survey about their usage of the manual including the reasons for any deviation from SOP. At the end, participants were paid and thanked for their involvement in this study.

\section{Results}

Statistical analyses were performed using SPSS. The results from the background knowledge test showed that participants assigned to different experimental conditions had different levels of knowledge related to operating hydraulic pump systems. Two-way ANOVA of the background knowledge score indicated a significant effect of manual version group assignment $(F(1,54)=10.581$, $\left.p=0.002, \eta^{2}=0.077\right)$, a significant effect of education level $\left(F(2,54)=25.286, p<0.001, \eta^{2}=0.367\right)$, as well as a significant interaction between the two $\left(F(2,54)=11.308, p<0.001, \eta^{2}=0.164\right)$. As a result, the background knowledge score was included in further analyses as a co-variant.

ANCOVA was conducted to examine the effects of manual type and education level on each of the dependent variables. Regarding task completion time, the effects were not significant for manual type $\left(F(1,53)=0.331, p=0.567, \eta^{2}=0.006\right)$, education level $\left(F(2,53)=0.333, p=0.718, \eta^{2}=0.012\right)$, and the interaction $\left(F(2,53)=0.496, p=0.612, \eta^{2}=0.018\right)$. Overall, participants completed each trial of production change task in $321 \mathrm{~s}$ on average $(S D=91 \mathrm{~s})$.

Regarding Percent Duration Within Bounds (PDWB), the effects were not significant for manual type $\left(F(1,53)=0.069, p=0.793, \eta^{2}=0.002\right)$, education level $\left(F(2,53)=0.089, p=0.915, \eta^{2}=0.003\right)$, and the interaction $\left(F(2,53)=0.313, p=0.732, \eta^{2}=0.011\right)$. Overall, participants achieved $43 \%$ PDWB $(S D=15 \%)$.

Regarding Adherence to Production Order Procedures (APOP), the results showed that manual type has a significant effect $\left(F(1,53)=6.571, p=0.013, \eta^{2}=0.099\right)$. The effects of education $(F(2,53)$ $\left.=1.991, p=0.147, \eta^{2}=0.060\right)$ and the interaction $\left(F(2,53)=1.368, p=0.263, \eta^{2}=0.041\right)$ were not significant. As shown in Figure 3, participants assigned to use the explanatory manual had significantly higher APOP $(M=68 \%, S D=39 \%)$ compared to those assigned the procedural manual $(M=44 \%$, $S D=44 \%$ ). Although there was no significant interaction effect, visual inspection of Figure 3 suggested that the difference between the two types of manuals might not be significant for participants from other faculties (non-engineering). A follow-up analysis was performed using independent samples $\mathrm{T}$ test (two-tailed). The result showed no significant difference between the explanatory and procedural manual conditions for non-engineering participants; $t(26)=0.444, p=0.66$.

Regarding the reasons why participants deviated from SOP, the most mentioned reason by $43 \%$ of the participants in the procedural manual condition was because they considered using their own methods as easier or better than the instructions provided in the manual, and which was followed by unintentional errors (mentioned by $33 \%$ ), such as slips and lapses. In the explanatory manual condition, the most mentioned reason was unintentional errors (mentioned by $53 \%$ ), and very few participants in this group thought it was easier or better using their own methods $(10 \%)$. 
Regarding Adherence to Wait Time (AWT), the effects were not significant for manual type $\left(F(1,53)=0.355, p=0.554, \eta^{2}=0.006\right)$, education level $\left(F(2,53)=0.526, p=0.594, \eta^{2}=0.019\right)$, and the interaction $\left(F(2,53)=0.399, p=0.673, \eta^{2}=0.014\right)$. Overall, participants achieved $62 \%$ AWT $(S D=29 \%)$.

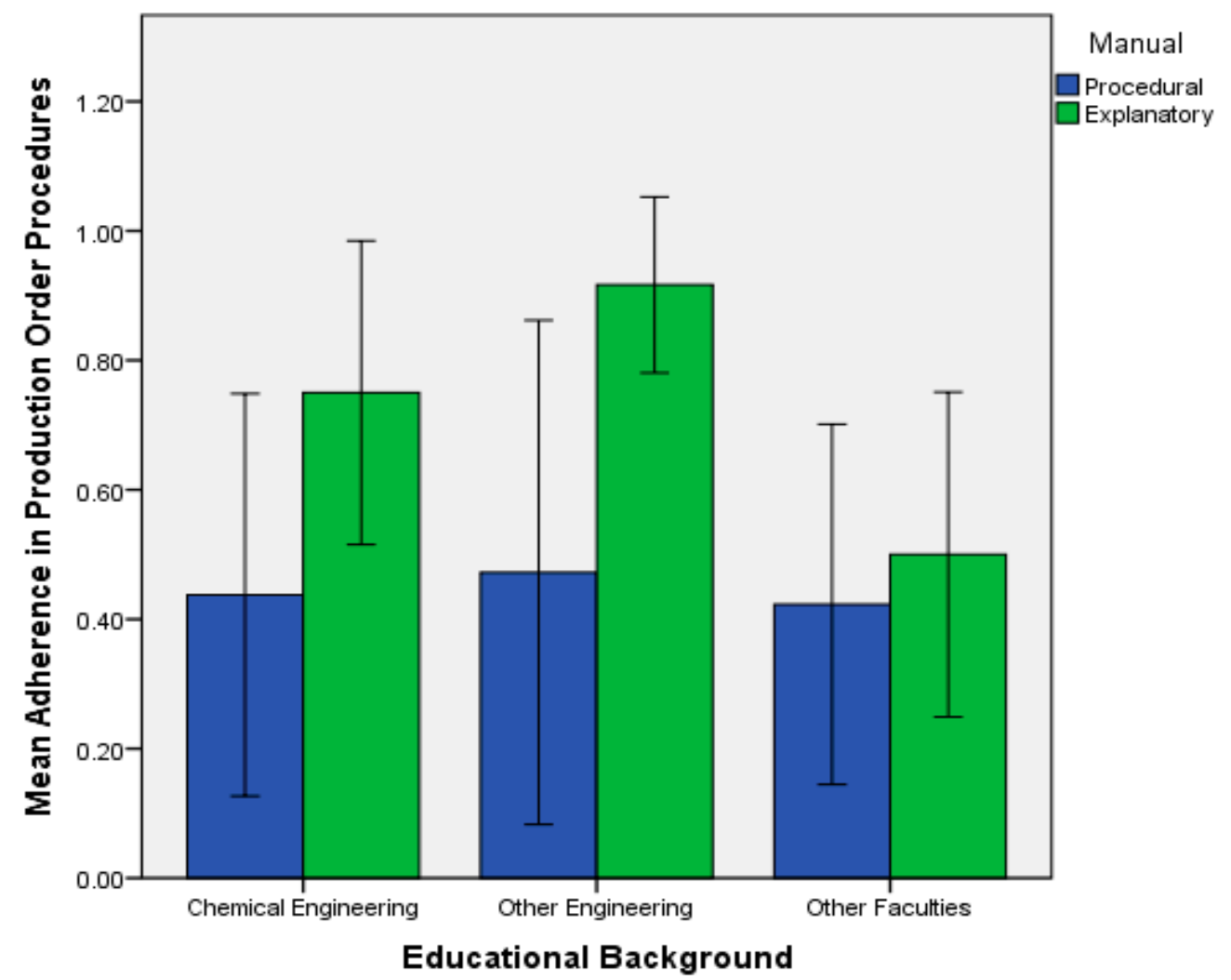

Error Bars: $95 \% \mathrm{Cl}$

Figure 3. Adherence to Production Order Procedures by manual type and education.

\section{Discussion}

The current study examined SOP manuals in routine operations where the tasks and steps can be clearly and precisely described. The results showed the benefit of using explanatory manual in comparison to procedural manual in terms of better adherence to operating procedures (i.e., APOP), while time performance and PDWB remained similar. This finding confirms that if employees are provided with a reason why they need to follow to certain procedural steps or sequences, they will be more likely to adhere than if they do not understand the importance for adhering and the consequence of not adhering. Although this idea is intuitive, it is interesting to find that a simple addition of the purposes and reasons in the manual can make a significant difference. Companies are sensitive to the cost associated with training. Implementing explanatory manual requires SOP designers to explicitly state their reasons behind each step but has minimal impact on the training procedures and training cost. With the help of explanatory manual, instructors can clearly and thoroughly explaining to new operators the purpose for performing certain steps that are essential to the operation. This is expected to help bridge the knowledge gap between SOP designers and operators. The current study showed the tendency for new operators to feel overwhelmed as they lack the experience and knowledge. If they do not clearly understand the necessity for certain procedures and its impact on the overall process and safety, they are likely to attempt to simplify the process or create their own methodologies following their intuition. As shown in the current results, the top reason for deviating from SOP when using the procedural manual was because participants considered using their own methods as easier or better. In the explanatory manual, however, the most mentioned reason was unintentional errors. The finding 
from the current study provided evidence to support the new approach of explanatory manual in SOP manual design.

Adherence to operating procedures indicates process safety, while performance measures such as task completion time focus on process efficiency. In this regard, no significant effect of manual type was found in the current study. It suggests that even when participants need to read more in the explanatory manual condition, their task time does not significantly increase.

The laboratory setup is a limitation of the current study. The setup was simplified in order to reduce learning requirements and allow participants without technical expertise to complete the tasks used this study. Due to the exploratory nature of this initial work using a hypothetical scenario, the design of SOP may not exactly follow general conventions for good procedure design. For example, some of the steps have multiple actions, which can be improved in future work. Industrial plants have much more complicated systems and SOPs. Future studies are needed to further examine the effect of explanatory manuals in actual industrial settings. Another limitation is the use of student trainees in a short duration of study. Recent research findings suggest that operators' level of experience affects their procedure usage [16]. Experienced operators, in comparison to novices, may find more issues with SOP and, therefore, be more likely to use their own methods and deviate from SOP. The effectiveness of explanatory manuals needs to be further examined in long-term studies and studies with experienced operators. Nevertheless, the controlled laboratory test in the current study serves as an initial step in examining this research question.

The results of the current study have significant implications for the process industry. Companies need effective and economic methods to improve operators' adherence to operating procedures. The current finding suggests that explanatory manuals can serve as such a method, which can be used both as learning materials in training and references in operation. We advocate the new approach of explanatory manual design. In addition to providing clear step-by-step instructions about how to do the tasks, designers are recommended to also give reasons why the steps should be closely followed. This also increases the requirement for designers because they must carefully examine their design of SOP and explain their knowledge to operators in plain language that matches the operators' level of knowledge and understanding. The results of Adherence to Production Order Procedures from the current study showed that participants with engineering education backgrounds benefited from the extra explanation in the explanatory manual, but the effect was not significant for participants from non-engineering background. It is possible that the non-engineering participants may have difficulty in comprehending the explanation. This finding not only emphasizes the need for operator selection but also highlights the challenge for SOP designers to clearly describe the procedures and their purposes with the consideration of operators' education background.

Although a significant effect of the type of manual on Adherence to Production Order Procedures was found, no significant effect was found for Adherence to Wait Time. A potential explanation may be that in this laboratory simulation scenario, participants may be reluctant to spend extra time waiting for hypothetical reasons. For example, two of the reasons introduced for a waiting procedure were to ensure all residual chemicals are flushed out and to improve washing quality. However, participants knew that only water was used in this test without any actual chemical. This is again a limitation of laboratory experimentation, which should be addressed in future work using actual industrial settings.

Knowing the reasons behind each step will help operators when they want to be creative and improve SOP. Operators' feedback to SOP manual design is important because operators may know more practical situations and constraints that are different from the designers' expectation and mental model. Operators should be encouraged to report new ideas to the designers so that the designers can gain the feedback and consider revising SOP based on new information. Through iteration and collaboration, the knowledge gap between designers and operators can be reduced, and SOP and process safety can be improved. Explanatory manuals are key to this new model of SOP design. It is important to note that the current results should be interpreted within routine operations and may not be applicable to complex domains such as healthcare because precisely prescribing SOP becomes 
difficult or impossible in such complex operations. Building upon the current study, future studies are needed to examine the effect of explanatory manual in actual industrial settings with workers as the participants.

\section{Conclusions}

In a controlled experiment of operating a hydraulic pump system that represented a routine operation in the process industry, participants' performance and adherence to operating procedures were measured and compared between the explanatory manual and the procedural manual conditions. The results showed that explanatory manuals had the benefit of increasing Adherence to Production Order Procedures, while time performance, Percent Duration within Bounds, and Adherence to Wait Time were not significantly affected. We advocate the use of explanatory manuals and the approach of explanatory SOP design that focus on bridging the knowledge gap between designers and operators. Explanatory manuals could be an effective and economic way to improve operators' adherence to operating procedures and should be further tested in industrial settings.

Author Contributions: Conceptualization, S.C. and A.E.; formal analysis, K.C.; funding acquisition, S.C.; investigation, K.C.; methodology, S.C. and A.E.; writing—original draft preparation, S.C. and K.C.; writing—review and editing, S.C. and A.E.

Funding: This work was partially funded by Natural Sciences and Engineering Research Council of Canada (NSERC) Discovery Grant to S.C. [RGPIN-2015-04134].

Conflicts of Interest: The authors declare no conflicts of interest. The funders had no role in the design of the study; in the collection, analyses, or interpretation of data; in the writing of the manuscript, or in the decision to publish the results.

\section{References}

1. Dien, Y. Safety and application of procedures, or how do they "have to use operating procedures in nuclear power plants?". Saf. Sci. 1998, 29, 179-187. [CrossRef]

2. Hollnagel, E.; Wears, R.L.; Braithwaite, J. From Safety-I to Safety-II: A White Paper; The Resilient Health Care Net; University of Southern Denmark: Sønderborg, Denmark; University of Florida: Gainesville, FL, USA; Macquarie University: Sydney, Australia, 2015; ISBN TBA.

3. Carvalho, P.V.; Dos Santos, I.L.; Vidal, M.C. Nuclear power plant shift supervisor's decision making during microincidents. Int. J. Ind. Ergon. 2005, 35, 619-644. [CrossRef]

4. Doak, S.; Assimakopoulos, D. Tacit knowledge: A needed addition to SOPs in a forensic science environment. Forensic Sci. Policy Manag. 2010, 1, 171-177. [CrossRef]

5. Chemical Safety Board. Investigation Report, Refinery Explosion and Fire; U.S. Chemical Safety and Hazard Investigation Board: Washington, DC, USA, 2007.

6. MacKenzie, C.; Holmstrom, D.; Kaszniak, M. Human Factors Analysis of the BP Texas City Refinery Explosion. In Proceedings of the Human Factors and Ergonomics Society Annual Meeting, Baltimore, MD, USA, 1-5 October 2007; Sage Publications Sage CA: Los Angeles, CA, USA, 2007; Volume 51, pp. 1444-1448.

7. International Atomic Energy Agency. Report on the Preliminary Fact Finding Mission Following the Accident at the Nuclear Fuel Processing Facility in Tokaimura, Japan; International Atomic Energy Agency: Vienna, Austria, 1999.

8. Stimpson, A.J.; Buinhas, L.S.; Bezek, S.; Boussemart, Y.; Cummings, M.L. A Model-Based Measure to Assess Operator Adherence to Procedures. In Proceedings of the Human Factors and Ergonomics Society Annual Meeting, Boston, MA, USA, 22-26 October 2012; Sage Publications Sage CA: Los Angeles, CA, USA, 2012; Volume 56, pp. 2452-2456.

9. Hopkins, A. Thinking about process safety indicators. Saf. Sci. 2009, 47, 460-465.

10. Chalk, S. Reducing human error: Leading industry collaborators outline top 10 best practices for human error reduction. BioPharm Int. 2012, 25, 1-3.

11. Akyar, I. Standard operating procedures (what are they good for?). In Latest Research into Quality Control; IntechOpen: London, UK, 2012. 
12. McKenzie, C. Creating a useful system manual: Documenting standard operating procedures ensures continuity. On Tap 2010, Winter, 27-30.

13. Environmental Protection Agency. Guidance for Preparing Standard Operating Procedures (SOPs); Environmental Protection Agency: Washington, DC, USA, 2007.

14. Sadoski, M.; Goetz, E.T.; Fritz, J.B. Impact of concreteness on comprehensibility, interest, and memory for text: Implications for dual coding theory and text design. J. Educ. Psychol. 1993, 85, 291. [CrossRef]

15. Kintsch, W. Text comprehension, memory, and learning. Am. Psychol. 1994, 49, 294. [CrossRef] [PubMed]

16. Sasangohar, F.; Peres, S.C.; Williams, J.P.; Smith, A.; Mannan, M.S. Investigating written procedures in process safety: Qualitative data analysis of interviews from high risk facilities. Process Saf. Environ. Prot. 2018, 113, 30-39. [CrossRef]

(C) 2019 by the authors. Licensee MDPI, Basel, Switzerland. This article is an open access article distributed under the terms and conditions of the Creative Commons Attribution (CC BY) license (http://creativecommons.org/licenses/by/4.0/). 